\title{
Frequency of Weight Gain and its Impact in Adults during Lockdown: A Cross- Sectional Study
}

\section{Uswah Bokhari, ${ }^{1}$ Uswah Shoaib, ${ }^{2}$ Sana Tariq, ${ }^{3}$ Sehar Khauteja Khan, ${ }^{4}$ Fatima Syed, ${ }^{5}$ Bushra Amin, ${ }^{6}$ Farhat Ijaz, ${ }^{7}$ Rana Khurram Aftab ${ }^{8}$}

\begin{abstract}
Objectives: The current lockdown in the country has led to a sedentary lifestyle because of a shift of daily activities online and a fear of contracting the illness leading to isolation inside homes; Hence an expected change in weight of the general population. This study aims to determine the frequency of weight gain and its impact in adults during the current lockdown of COVID-19.

Methods: Participants selected from the ages of 18-25 from different universities of Pakistan were sent questionnaires via WhatsApp, posted online using Microsoft Forms. A total of 157 responses were attained.

Results: Among the 157 participants the average weight before the lockdown was $61.95 \mathrm{kgs}$ and after the lockdown was $64.99 \mathrm{kgs}$ so there was an increase of $3.016 \mathrm{kgs}$ whereas $18.5 \%$ of the sample maintained their weight and $37.6 \%$ were reported to have lost weight. The results also depict a $1.6 \%$ increase in impairment of body image.

Conclusion: We concluded that the Lockdown due to the COVID-19 pandemic has caused a gain in the weight and lowered self-esteem with regard to body image of most young adults in Pakistan due to many factors including higher food intake, lack of exercise and also emotional distress.

Key words: Obesity, Weight gain, Lockdown, Body Image.

How to Cite: Bokhar U, Shoaib U, Tariq S, Khan S.K, Syed F, Amin B, Ijaz F, Aftab R.K. Frequency of weight gain and its impact in adults during lockdown: a cross sectional study. Esculapio.2020;16(04):110-115.
\end{abstract}

DOI: https://doi.org/10.51273/esc20.2516424

\section{Introduction}

$\mathrm{O}$ n March 11, 2020 WHO Director General declared COVID-19 a global pandemic, the first ever pandemic caused by the coronavirus species. ${ }^{1}$ As governments, started to lockdown there countries, the stress and anxiety among the populations raised, as Brooks et al. found in their review a positive correlation of increased mental health problems during this pandemic. The factors leading to this rise include the long duration of isolation, fear of infection, inade-

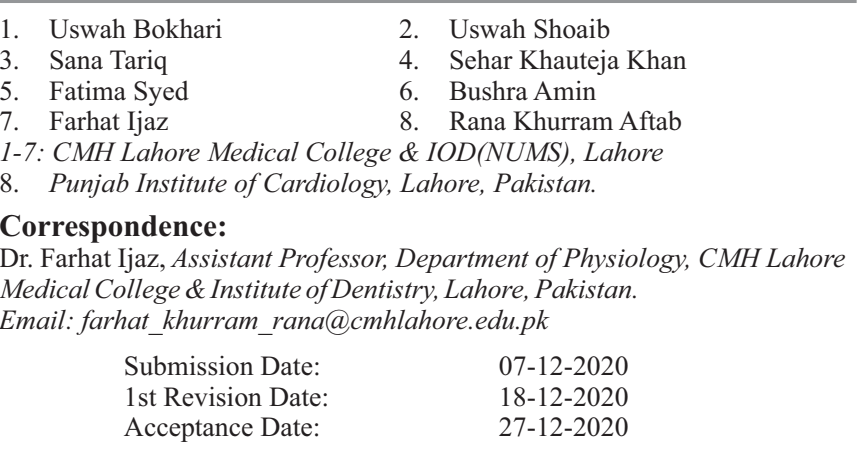

quate supplies, boredom and frustration surrounding financial situations due increasing unemployment. ${ }^{2}$ The article published by Zachery et al. May 2020, found that $22 \%$ of the subjects gained 5-10 pounds during self-isolation as they spent $20-24 \mathrm{hrs}$ inside the house. ${ }^{3}$ Furthermore, this study also found that a significantly higher percentage of people that gained weight were eating in response to stress or snacking after dinner. $^{3}$

As the uncertainty surrounding and duration of the pandemic increases, people tend to make unhealthier lifestyle choices. People tend to choose foods with longer shelf life that are highly processed and rich in calories. ${ }^{4}$ This not only affects adults but also children. As schools, workplaces and gyms closed down, opportunities for physical activity also declined. Furthermore, people also have an unhealthier sleeping pattern during holidays. All of these factors i.e. unhealthy diet, lack of physical activity and poor sleeping habits lead to a heightened risk of obesity. ${ }^{3}$ 
As it is well known, obesity is linked to heightened morbidity and mortality. Obesity causes a low-grade systemic inflammation that leads to the development of metabolic dysfunction. ${ }^{5}$ According to Bary et al. dysfunctional subcutaneous fat deposition lead to increased free fatty acids and decreases triglycerides which in turn causes ectopic fat deposition. This lipid deposition in various systems of the body lead to cardiovascular disease, diabetes mellitus and dyslipidemia. On the other hand there is increase in cytokines release and angiotensin which causes hypertension. ${ }^{6}$ Furthermore, there is an increased risk of breast cancer, endometrial cancer and other forms of cancer. $^{7-9}$ Obesity also modifies the immune responses of the body, making the immune system more vulnerable to infections and less responsive to vaccinations, antivirals and antimicrobial therapies. ${ }^{10}$ It has with higher incidences of respiratory tract infections as well as rhinitis, sinusitis and pharyngitis/ laryngitis in obese individuals. ${ }^{11}$ A greater incidence of bronchitis, pneumonia and influenza like illness has also been reported in obese individuals. ${ }^{12-14}$ In addition, according to Lee et al. and Delvin et al. there is a rise in mental health problems as obese people are more prone to weight based bullying, body image issues, depression and eating disorders. ${ }^{15,16}$ Keeping in view all these factors, this study was aimed to determine frequency of weight gain and its impact in adults during the current lockdown of COVID-19.

\section{Methods}

After approval by the ethical committee of $\mathrm{CMH}$ LMC \& IOD, participants both male and female, age group of 18-25years, belonging to different universities of Pakistan were offered to be enrolled in the study. The sample size was calculated to be 157 using the Rao soft formula with $95 \%$ confidence interval and $5 \%$ margin error. The population size used was calculated by using values of Zachary et al. ${ }^{2}$

Questionaries' were administered online using Microsoft Forms by sending the link for the form to WhatsApp groups. The questionnaires were adapted using Impact of Weight on Quality of Life (IWQOL) ${ }^{17}$ and Body Image Questionnaire (BIQ). ${ }^{18}$ The questions designed included questions from four areas: physical function, self-esteem, public distress and body image, respectively. Question 1 to 6 collected biodata of the participants. Question 7-16 were adapted from the IWQOL. Each question was rated on a three-point Likert scale with 'Never' scoring 0 points, 'Sometimes' scoring 3 points and 'All the time' scoring 5 points. The scores are additive and higher scores mean lower impact of weight gain on physical function, self-esteem, public distress. Question 17 21 were adapted from the Body Image Questionnaire (BIQ). ${ }^{18}$ This part consist of 5 questions related to body image. Each item for this part was scored from 1 i.e., impaired to 5 i.e., most impaired. The total score was achieved by summing all scores. The total scores ranged from 6-30 with a higher score reflecting greater impairment of body image. All data was analyzed using SPSS software (version 26; IBM). Results were presented in frequency and percentages. Chi- square test was used for comparison of categorical variables. $\mathrm{P}$ value $<0.05$ was statistically significant.

\section{Results}

One hundred and fifty-seven people completed the survey of which 109 were females and 48 males. The average weight before lockdown was $61.95 \mathrm{kgs}$ and after lock down was $64.966 \mathrm{kgs}$, an increase of 3.016 kgs. Stable weight was maintained by $18.5 \%$ of the sample while $37.6 \%$ and $11.5 \%$ reported to have gained less than 5 kilograms and more than 5 kilograms respectively. This data is presented in Figure 1.

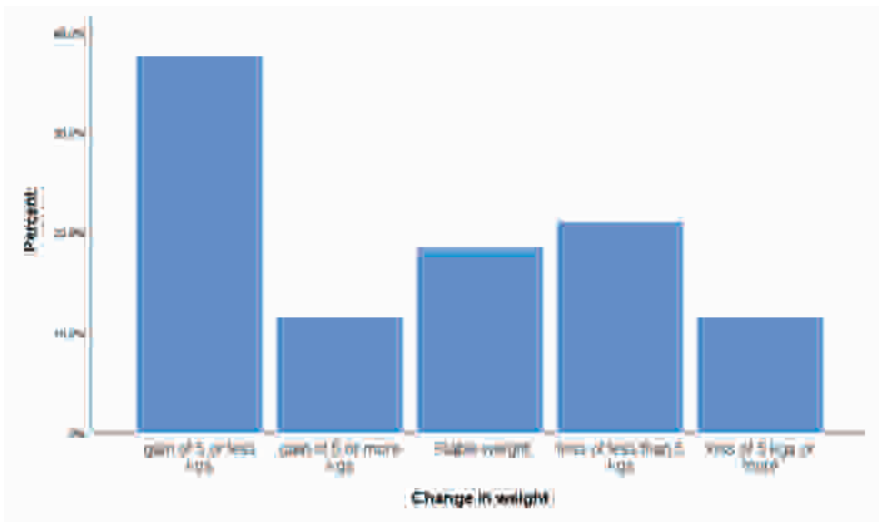

Figure 1: Change in Weight by Percentage of Population

The average score for part 1 of the survey assessing quality of life was 12.185 before lockdown and 12.86 after lockdown (Table 1). The increase in this score shows that the overall quality of life worsened during lockdown however this difference is not significantly high. As represented by Table 1, physical function declined in two variables, trouble with moderate exercise i.e., trouble climbing stairs/running with a P- 
value of 0.033 and trouble with stiff joints i.e. I am troubled by painful or stiff joints with a P-value of 0.002 . The area of self-esteem also showed significant $\mathrm{p}$-values for the variables 'Because of my weight, I am afraid of being rejected' and 'Because of my weight, I avoid looking in mirrors or seeing myself in photographs' with p-values of 0.04 and 0.033 respectively. The P-value was also significant for the variable concerning the area of work i.e. trouble getting things accomplished or meeting my responsibilities (0.02). The average score for part 2 of the survey assessing impairment in body image was 12.796 before lock-down and 13.701 after lockdown (Table 1). This demonstrates that there has been an increase in the likelihood of impaired body image. Assessment of impairment of body image demonstrate an

Table 1: Scores and Standard Deviation for Assessment of Quality of Life and body Image

\begin{tabular}{lcccc}
\hline & \multicolumn{2}{c}{$\begin{array}{c}\text { Assessment of } \\
\text { quality of life }\end{array}$} & \multicolumn{2}{c}{$\begin{array}{c}\text { Assessment of } \\
\text { body image }\end{array}$} \\
\hline Average Score & Mean & Std & Mean & Std \\
Before lockdown & 12.1847 & 3.2420 & 12.7962 & 4.0884 \\
After lockdown & 12.8599 & 3.8886 & 13.7006 & 4.8417 \\
\hline
\end{tabular}

increased impairment in some variables.

Table 2 represents the variables with significant $\mathrm{p}$ values when populations were compared according to their change in weight. People were significantly selfconscious because of their weight after lockdown with a p-value of 0.026. Experience of being ridiculed, teased, or receiving unwanted attention increased in population with gain in weight, while it declined in those that lost weight(0.06).

For the area of body image after lockdown, p-value was significant for "How noticeable do you feel your weight was/is/will be to a stranger(0.043). From the population with gain of $5 \mathrm{kgs}$ or more 4 people reported feeling that their weight was markedly noticeable to a stranger; however, this number doubled to 8 after lockdown.

\section{Discussion}

This cross-sectional study shows the association between weight gain (obesity) and lockdown during the Covid-19 pandemic in Pakistan. The population studied ranged from 18-25 years with mostly students who stayed home from Mid-March of 2020 to 15th September, 2020. The results how weight gain affected the physical and mental health of people. $49.1 \%$ of the participants gained weight and the rest of the $50.9 \%$ either had stable weight or had weight loss. We have focused on the effects of weight gain which may be due to poor physical activity as weren't allowed to go outside and many people prefer gyms/outdoors over home exercises, increased screen time, high fatty diet, and less vegetables. Another important reason was that the lockdown period was associated with a higher level of stress and people resorted towards "stress eating" as a coping mechanism as set by prior research of Zachary $2020 .^{3}$ The most common side effect of weight gain was reduction in physical activities. Of those who gained weight 12 participants had trouble performing daily activities, 8 had difficulty in climbing stairs and 14 reported and an increase in joint pain. This showed that quite a few numbers of participants had side effects of obesity on their health. This can lead to complications later, if obesity is not controlled, such as Diabetes, PCOS in young women, atherosclerosis etc. An article reported that women were at a greater risk of gaining weight due to stressrelated eating. ${ }^{19}$ This also brings attention to a relatively ignored population during this pandemic; women with PCOS, the most frequent endocrine disorder in women of reproductive age. ${ }^{20}$ According to Barber et al. Even though postprandial thermogenesis was significantly lower in women with PCOS, it cannot be linked to weight gain as the resting metabolic rates remain similar to those without PCOS. ${ }^{21}$ However, as established by Deeks et al. PCOS is a significant contributor to anxiety and depression. ${ }^{22}$ Anxiety and depression, however have are linked to higher BMI, especially in women. ${ }^{23}$ With the rise in anxiety due to the pandemic, there may be an increased risk of obesity in women, especially those with an underlying metabolic disorder like PCOS. Ultimately, this may attribute towards the fact that the amount of weight gain might have led to increased levels of inflammatory mediators like TNF,IL-1,IL-6, resulting in osteoarthritis as mentioned previously by Vincent $\mathrm{HK} .{ }^{24}$ The problem of osteoarthritis linked with obesity was more commonly seen in women; it affected the larger stabilizing joints mostly like knee, back and hip, that are important in performing physical activities like climbing stairs as presented also by Elizabeth $\mathrm{M}$ Badley in 2020. ${ }^{25}$

In our society particularly the South Asian society weight gain comes with a social stigma that has a considerable amount of negative effect on the mental 


\section{Physical Function}

Because of my

weight, I have trouble tying my shoes, putting on/taking off clothes, crossing my legs etc.

I am troubled by painful or stiff joints

\section{Self esteem}

Because of my weight, I am selfconscious

Because of my weight, I avoid looking in mirrors or seeing myself in photographs

Because of my weight, I experience ridicule, teasing, or unwanted attention

Body Image

How noticeable do you feel your weight was/is/will be to a stranger (if you do not camouflage yourself e.g. with clothes and/or makeup) and the feature has not been pointed out to them?

How much do you feel your weight/body shape is the most important aspect of who you are?

How much do you feel your
weight/body shape is the most
important aspect of who you are?

How much do you feel your weight/body shape is the most important aspect of who you are?

\begin{tabular}{|c|c|c|}
$\begin{array}{c}\text { Population } \\
\text { with gain } \\
\text { of less than }\end{array}$ & $\begin{array}{c}\text { Population } \\
\text { with gain } \\
\text { of } 5 \text { kgs or } \\
\text { mgs }\end{array}$ & $\begin{array}{c}\text { Stable } \\
\text { weight }\end{array}$ \\
more
\end{tabular} \mid

\begin{tabular}{|c|c|c}
$\begin{array}{c}\text { Population } \\
\text { with loss } \\
\text { of less }\end{array}$ & $\begin{array}{c}\text { Population } \\
\text { with loss } \\
\text { of 5kgs or } \\
\text { than 5kgs }\end{array}$ & $\begin{array}{c}\text { P- } \\
\text { value }\end{array}$ \\
more &
\end{tabular}

\begin{tabular}{|c|l|r} 
Before & Never & 57 \\
Sometimes & 2 \\
& Always & \\
Befer & Never & 48 \\
& Sometimes & 1 \\
Always & 1 \\
Aftere & Sometimes & 4 \\
& Always & 5 \\
& Never & 35 \\
& Sometimes & 24 \\
& Always &
\end{tabular}

\begin{tabular}{c|c}
57 & 16 \\
2 & \\
- & \\
48 & \\
10 & \\
1 & 8 \\
44 & \\
10 & 10 \\
5 & 6 \\
35 & \\
24 & \\
- &
\end{tabular}

\begin{tabular}{c|c|c|c}
16 & 27 & 29 & 12 \\
2 & 2 & 2 & 6 \\
- & - & - & - \\
8 & 27 & 30 & 16 \\
8 & 1 & 3 & 2 \\
2 & 1 & - & - \\
10 & 21 & 20 & 8 \\
6 & 5 & 9 & 8 \\
2 & 3 & 4 & 2 \\
6 & 19 & 19 & 13 \\
9 & 10 & 12 & 5 \\
3 & - & 12 & -
\end{tabular}

0.021

16

0.0001

$-$

8

8

0.312

13

5

0.018

\begin{tabular}{|c|l|c} 
Before & Never & 26 \\
After & Sometimes & 23 \\
& Always & 1 \\
Bever & Sometimes & 23 \\
& Always & 1 \\
After & Never & 49 \\
& Sometimes & 8 \\
Before & Always & 2 \\
& Never & 42 \\
Always & Sometimes & 16 \\
& Always & 1 \\
Alever & Sometimes & 13 \\
& Always & 2 \\
Never & Sometimes & 39 \\
& Always & 2
\end{tabular}

\begin{tabular}{c|}
26 \\
23 \\
10 \\
23 \\
22 \\
14 \\
49 \\
8 \\
2 \\
42 \\
16 \\
1 \\
44 \\
13 \\
2 \\
39 \\
18 \\
2
\end{tabular}

\begin{tabular}{c|c}
8 & 16 \\
7 & 8 \\
3 & 5 \\
4 & 16 \\
6 & 10 \\
8 & 3 \\
11 & 23 \\
6 & 23 \\
1 & - \\
6 & 25 \\
8 & 5 \\
4 & 1 \\
8 & 18 \\
10 & 10 \\
18 & 1 \\
5 & 19 \\
11 & 8 \\
2 & 2
\end{tabular}

\section{1}

17

\begin{tabular}{c|c}
6 & \\
10 & 0.874 \\
2 & \\
8 & \\
8 & 0.026 \\
5 & \\
10 & \\
7 & 0.108 \\
1 & \\
13 & \\
15 & 0.001 \\
- & \\
5 & \\
10 & 0.002 \\
3 & \\
11 & \\
5 & 0.066 \\
2 &
\end{tabular}

\begin{tabular}{|c|c|c|c|c|c|c|c|}
\hline \multirow{5}{*}{ Before } & Not at all & 25 & 5 & 11 & 12 & 1 & \multirow{5}{*}{0.015} \\
\hline & Slightly & 3 & 6 & 7 & 12 & 6 & \\
\hline & Moderately & 9 & 3 & 5 & 8 & 6 & \\
\hline & Markedly & 2 & 4 & 6 & 1 & 4 & \\
\hline & Very & 3 & - & - & - & - & \\
\hline \multirow{5}{*}{ After } & Not at all & 18 & 2 & 11 & 10 & 2 & \multirow{5}{*}{0.043} \\
\hline & Slightly & 19 & 4 & 7 & 12 & 8 & \\
\hline & Moderately & 14 & 3 & 5 & 8 & 5 & \\
\hline & Markedly & 4 & 8 & 5 & 2 & 1 & \\
\hline & Very & 4 & 1 & 1 & 1 & 2 & \\
\hline \multirow{5}{*}{ Before } & Not at all & 8 & 1 & 6 & 5 & 1 & \multirow{5}{*}{0.964} \\
\hline & Slightly & 16 & 5 & 7 & 10 & 6 & \\
\hline & Moderately & 20 & 10 & 7 & 10 & 6 & \\
\hline & Mostly & 13 & 2 & 7 & 8 & 5 & \\
\hline & Totally & 2 & 18 & 2 & - & - & \\
\hline \multirow{5}{*}{ Before } & Not at all & 8 & 1 & 6 & 5 & 1 & \multirow{5}{*}{0.964} \\
\hline & Slightly & 16 & 5 & 7 & 10 & 6 & \\
\hline & Moderately & 20 & 10 & 7 & 10 & 6 & \\
\hline & Mostly & 13 & 2 & 7 & 8 & 5 & \\
\hline & Totally & 2 & 18 & 2 & - & - & \\
\hline \multirow{5}{*}{ After } & Not at all & 10 & 1 & 7 & 4 & 2 & \multirow{5}{*}{0.311} \\
\hline & Slightly & 13 & 3 & 6 & 9 & 5 & \\
\hline & Moderately & 22 & 6 & 8 & 12 & 7 & \\
\hline & Mostly & 10 & 5 & 6 & 7 & 4 & \\
\hline & Totally & 4 & 3 & 2 & 1 & - & \\
\hline
\end{tabular}


health of slightly obese and obese people. People tend to lose self-confidence, self-esteem and start avoiding their own body image. This has been highlighted in our results as well. Before lockdown $60.1 \%$ people were self-conscious about their weight and $61.7 \%$ were self-conscious after lockdown. More number of people feared social rejection based on their body weight after lockdown than before lockdown. This led them to avoid looking at their body image in mirrors (self- avoidance). Other contributory factors were peer victimization, unwanted teasing, societal pressure as given in prior research of Lowry in $2007^{26}$ and has also been shown in our results. There was a gradual increase in number of young adults who were facing teasing and ridiculing due to weight gain after lockdown, whereas it decreased for people who lost weight after lockdown which depicts the strength of peer pressure on overweight individuals.

We lived in a difficult time during the Covid-19 pandemic with a lot of people reporting mental health issues they faced during lockdown and post lockdown. Weight gain could be a contributing factor. The limitations of our study include the questionnaires being sent online there might be a chance that these were not solved by the individuals themselves which may lead to ambiguous results. A proper identification procedure must have been added to avoid such discrepancy. A broader spectrum of age groups should have been used to get an accurate idea about how the weight altered within individuals. Based upon the number of responses, especially the huger share contributed by adults with less than $5 \mathrm{~kg}$ gain of weight, it may be suggested that they immediately switch to less fatty diet and observe healthier activities to cover up this short range of weight gain easily. Another limitation that the female responses were not segregated during data collection for the variables and hence the ratio of young females being affected by stiff joints, cannot be deciphered.

\section{Conclusion}

In conclusion, it is seen that the community-wide quarantine during COVID-19 pandemic has caused weight gain in adults. This weight gain has occurred due to availability of calorific processed foods, lack of physical activity, emotional distress and overall a sedentary lifestyle that seem to dominate the COVID19 isolation. Such weight gain is not only deleterious for those who are already overweight, obese, immu- nocompromised or suffer from severe mental illnesses but also those who were of normal weight pre lockdown, immunocompetent neurotypicals. Our research clearly depicts the substantial changes in weight i.e., an increase of $3.016 \mathrm{~kg}$ weight on average, post lockdown and an increase of $1.6 \%$ in impairment of body image among adults falling in 18-25 age group. With above mentioned results, there's no doubt that covid-19 isolation has not only led to stagnancy causing weight gain in young adults but also low self-esteem and negative body image ultimately giving rise to unhealthy individuals with mental health issues. So, the question arises are we really ready to deal with a low esteemed debilitating generation when the pandemic is finally over?

\section{Conflict of Interest: $\quad$ None}

\section{References}

1. Coronavirus (COVID-19) events as they happen. Accessed July 26, 2020. https://www.who.int/ emergencies/ diseases/novel-coronavirus-2019/eventsas-they-happen

2. Brooks SK, Webster RK, Smith LE, et al. The psychological impact of quarantine and how to reduce it: rapid review of the evidence. Lancet. 2020; 395 (10227):912-920. doi:10.1016/S0140-6736(20) 30460-8

3. Zachary Z, Brianna F, Brianna L, et al. Self-quarantine and weight gain related risk factors during the COVID-19 pandemic. Obes Res Clin Pract. Published online 2020. doi:10.1016/j.orcp. 2020.05.004

4. Rundle AG, Park Y, Herbstman JB, Kinsey EW, Wang YC. COVID-19-Related School Closings and Risk of Weight Gain Among Children. Obesity. 2020; 28(6):1008-1009. doi:10.1002/oby.22813

5. Muscogiuri G, Pugliese G, Barrea L, Savastano S, Colao A. Commentary: Obesity: The "Achilles heel" for COVID-19? Metabolism. 2020;108:154251. doi:10.1016/j.metabol.2020.154251

6. Bray GA, Heisel WE, Afshin A, et al. The science of obesity management: An endocrine society scientific statement. Endocr Rev. 2018;39(2):79-132. doi: 10. 1210/er.2017-00253

7. Rosner B, Eliassen AH, Toriola AT, et al. Short-term weight gain and breast cancer risk by hormone receptor classification among pre- and postmenopausal women. Breast Cancer Res Treat. 2015;150(3):643653. doi:10.1007/s10549-015-3344-0

8. Aune D, Rosenblatt DAN, Chan DSM, et al. Anthropometric factors and ovarian cancer risk: A 
systematic review and nonlinear dose-response metaanalysis of prospective studies. Int J Cancer. 2015; 136(8):1888-1898. doi:10.1002/ijc.29207

9. Calle EE, Rodriguez C, Walker-Thurmond K, Thun MJ. Overweight, obesity, and mortality from cancer in a prospectively studied cohort of U.S. Adults. N Engl J Med. 2003;348(17):1625-1638. doi: 10.1056/ NEJMoa021423

10. Muscogiuri G, Pugliese G, Barrea L, Savastano S, Colao A. Obesity: The "Achilles heel" for COVID19? Metabolism. 2020;108. doi:10.1016/ j.metabol. 2020. 154251

11. Schwartz MW, Seeley RJ, Zeltser LM, et al. Obesity pathogenesis: An endocrine society scientific statement. Endocr Rev.2017;38(4):267-296.doi:10. 1210/ ER.2017-00111

12. Lee YL, Chen Y-C, Chen Y-A. Obesity and the occurrence of bronchitis in adolescents. Obesity. 2013;21(1):E149-E153. doi:10.1002/oby.20262

13. Almirall J, Bolíbar I, Serra-Prat M, et al. New evidence of risk factors for community-acquired pneumonia: A population-based study. Eur Respir J. 2008; 31(6):1274-1284. doi:10.1183/09031936.00095807

14. Cocoros NM, Lash TL, Demaria A, Klompas M. Obesity as a risk factor for severe influenza-like illness. Influenza Other Respi Viruses. 2014;8(1):2532. doi:10.1111/irv.12156

15. Lee BY, Bartsch SM, Mui Y, Haidari LA, Spiker ML, Gittelsohn J. A systems approach to obesity. doi: 10. 1093/ nutrit/nuw049

16. Devlin MJ, Yanovski SZ, Wilson GT. Obesity: What mental health professionals need to know. Am J Psychiatry. 2000;157(6):854-866. doi:10.1176/appi. ajp. 157.6.854

17. Kolotkin RL, Head S, Hamilton M, Tse CJ. Assessing Impact of Weight on Quality of Life. Obes Res. 1995; 3(1):49-56. doi:10.1002/j.1550-8528.1995. tb00120. $\mathrm{x}$

18. King's College London - Body Image Questionnaires. Accessed July 27, 2020. https:// www.kcl.ac. uk/ioppn/depts/psychology/research/ResearchGrou pings/CADAT/Research/Body-Image-Questionnaires

19. Mattioli AV, Pinti M, Farinetti A, Nasi M. Obesity risk during collective quarantine for the COVID-19 epidemic. Obes Med. Published online June 9, 2020:100263. doi:10.1016/j.obmed.2020.100263

20. Kyrou I, Karteris E, Robbins T, Chatha K, Drenos F, Randeva HS. Polycystic ovary syndrome (PCOS) and COVID-19: an overlooked female patient population at potentially higher risk during the COVID-19 pandemic. BMC Med. 2020;18(1):220. doi:10.1186/s12916-020-01697-5

21. Barber TM, Hanson P, Weickert MO, Franks S. Obesity and Polycystic Ovary Syndrome: Implications for Pathogenesis and Novel Management Strategies. Clin Med Insights Reprod Heal. 2019;13:117955811987404. doi: 10.1177/ 1179558119874042

22. Deeks AA, Gibson-Helm ME, Paul E, Teede HJ. Is having polycystic ovary syndrome a predictor of poor psychological function including anxiety and depression? doi:10.1093/humrep/der071

23. Anderson SE, Cohen P, Naumova EN, Must A. Association of depression and anxiety disorders with weight change in a prospective community-based study of children followed up into adulthood. Arch Pediatr Adolesc Med. 2006;160(3):285-291. doi: 10.1001/archpedi.160.3.285

24. Vincent H, Heywood K, Connelly J, Hurley R. Obesity and Weight Loss in the Treatment and Prevention of Osteoarthritis. PM R. 2012;4:S59-67. doi: 10.1016/j.pmrj.2012.01.005

25. Badley EM, Wilfong JM, Yip C, Millstone DB, Perruccio A V. The contribution of age and obesity to the number of painful joint sites in individuals reporting osteoarthritis: a population-based study. Rheumatology (Oxford). 2020;59(11):3350-3357. doi: 10. 1093/ rheumatology/keaa138

26. Lowry KW, Sallinen BJ, Janicke DM. The effects of weight management programs on self-esteem in pediatric overweight populations. J Pediatr Psychol. 2007;32(10):1179-1195. doi:10.1093/jpepsy/jsm048

\section{Author's Contribution}

BU: Literature search, study design \& concept, questionaire design, data collection

SU: Literature search, study design \& concept, questionaire design, data collection, data analysis

TS, KKS, SF: Literature search, study design \& concept, questionaire design, data interpretation, drafting

AB: Data analysis, data interpretation, drafting

IF: Overall supervision, drafting, revision \& final approval

AKR: Data analysis, data interpretation, drafting 